\title{
Comparison of three-dimensional and two-dimensional statistical mechanics of shear layers for flow between two parallel plates
}

Received: 13 November 2011 / Accepted: 5 May 2012 / Published online: 23 May 2012

(C) The Author(s) 2012. This article is published with open access at Springerlink.com

\begin{abstract}
It is shown that the averaged velocity profiles predicted by statistical mechanics of point vortices and statistical mechanics of vortex lines are practically indistinguishable for a shear flow between two parallel walls.
\end{abstract}

Keywords Vortex dynamics · Hamiltonian systems · Turbulence $\cdot$ Boundary layer

\section{Introduction}

It is known that statistical mechanics of point vortices describes surprisingly well the averaged velocity profiles of self-similar mixing layer. After development of statistical mechanics of vortex lines [2-6], where a vortex does not remain straight and is allowed to take wavy shapes in the course of motion, it appeared a concern that the above-mentioned feature of statistical mechanics of point vortices can be lost in three-dimensional theory. In this paper, we show that this is not the case: the averaged velocity profiles predicted by statistical mechanics of point vortices and statistical mechanics of vortex lines are practically indistinguishable for a shear flow between two parallel walls.

\section{Averaged equations for flow between two plates}

Consider a flow of ideal incompressible fluid between two parallel walls, $y$ is the coordinate normal to the walls, $-h \leq y \leq h, 2 h$-the distance between the walls. The flow is modeled by motion of a large number of vortices (Fig. 1). Flow is periodic in $x$-direction and $z$-direction. In the limit of infinite period in $z$-direction, the averaged velocity is parallel to the walls, does not depend on $x$ and has the only non-zero component, $u=u(y)$. Assuming that vortices have the same intensity and the total discharge is zero, one obtains for the stream function of the averaged flow, $\psi=\psi(y)(u \equiv \mathrm{d} \psi / \mathrm{d} y)$, the equation

$$
\frac{\mathrm{d}^{2}}{\mathrm{~d} y^{2}} \psi=-\sigma f(y),\left.\quad \frac{\mathrm{d}}{\mathrm{d} y} \psi\right|_{ \pm h}= \pm U
$$

Communicated by Samuel Forest.

L. Shirkov $(\varangle)$

Department of Chemistry, University of Warsaw, Warsaw, Poland

E-mail: leonid.shirkov@gmail.com

V. Berdichevsky

Mechanical Engineering, Wayne State University, Detroit, MI 48202, USA

E-mail: vberd@eng.wayne.edu 


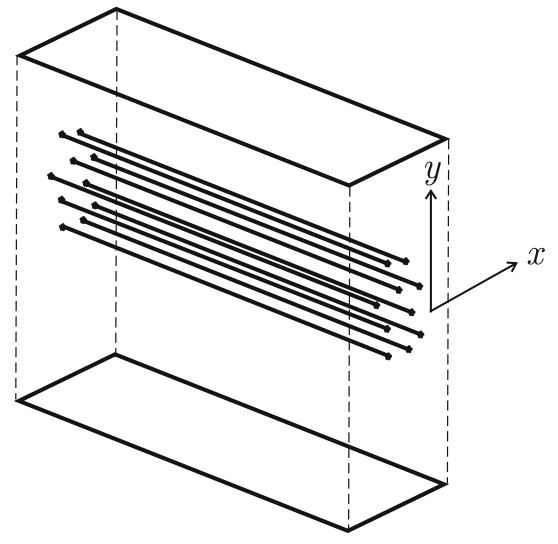

a

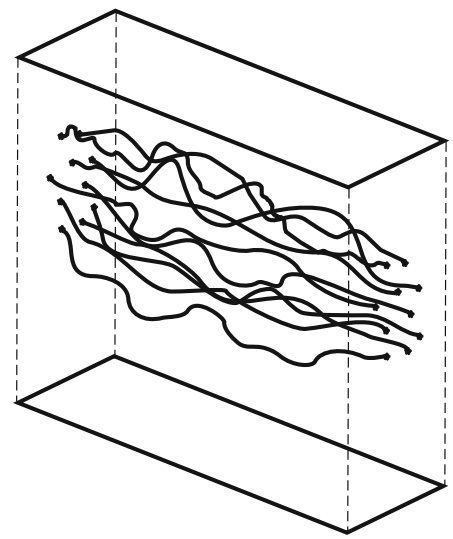

b

Fig. 1 Two-dimensional flow of point vortices (a) and three-dimensional flow of vortex lines (b)

where $f(y)$ is the probability to find a vortex at the point $y, \sigma$ is the total vorticity per unit length in $x$-direction, $\sigma=-2 U$. In the case of point vortices (Fig. 1a), [7,8]

$$
f(y)=\frac{\mathrm{e}^{-\beta \sigma \psi(y)}}{\int_{-h}^{h} \mathrm{e}^{-\beta \sigma \psi\left(y^{\prime}\right)} \mathrm{d}^{2} y^{\prime}},
$$

and Eqs. (1), (2) form a closed system of equations. This system can be solved analytically. Parameter $\beta$ has the meaning of inverse temperature of vortex motion. It is determined by the initial energy of turbulent flow.

In the case of deforming vortex lines (Fig. 1b), $f(y)$ is expressed through the solutions of the eigenvalue problem, $[4,5]$

$$
\Delta \varphi-\beta \sigma \psi \varphi=-\lambda \varphi,\left.\quad \frac{\mathrm{d}}{\mathrm{d} y} \varphi\right|_{ \pm h}=0,
$$

$\lambda$ being the minimum eigenvalue. Similarly to quantum mechanics, $f(y)$ is proportional to the squared solution of the eigenvalue problem:

$$
f(y)=\frac{\varphi^{2}(y)}{\int_{-h}^{h} \varphi^{2}(y) \mathrm{d} y^{\prime}} .
$$

We aim to compare solutions of Eqs. (1), (2) and (1), (3), (4).

\section{Results and discussion}

There is no reason to expect that the velocity profiles found from the two quite different system of Eqs. (1)-(2) and Eqs. (1), (3), (4), coincide. Nevertheless, this turns out to be the case: the velocity profiles are practically indistinguishable. More precisely: for each $\beta$ from " $3 D$ problem" Eqs. (1), (3), (4), there is $\beta$ from " $2 D$ problem" Eqs. (1)-(2) for which the velocity profiles practically coincide.

System of Eqs. (1)-(2) admits an analytical solution:

$$
\psi=-\frac{1}{U \beta} \ln \cosh (C y),
$$

where the constant $C$ is determined from the boundary condition,

$$
U+\frac{C}{U \beta} \tanh (C h)=0 .
$$



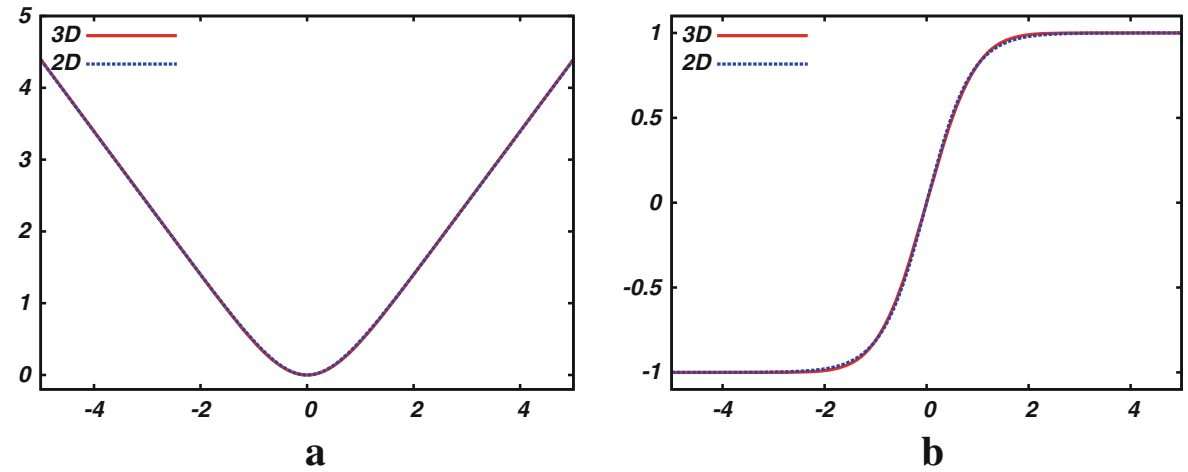

Fig. 2 Stream function (a) and velocity (b) profiles for $\beta_{3 D}=-1.0$ and $\beta_{2 D}=-1.1374$

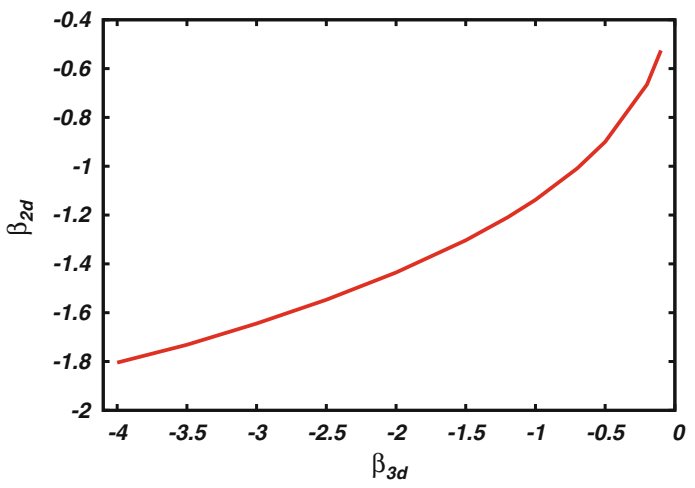

Fig. 3 Two-dimensional inverse temperature $\beta_{2 D}$ versus three-dimensional inverse temperature $\beta_{3 D}$

For $h=\infty, C=-U^{2} \beta$ and Eq. (5) becomes:

$$
\psi=-\frac{1}{U \beta} \ln \cosh \left(U^{2} \beta y\right) .
$$

All parameters of the flow can be normalized with respect to the wall velocity: $\psi \rightarrow \psi / U, \sigma \rightarrow \sigma / U$ and $\beta \rightarrow \beta U^{2}$. The dimension of the normalized $\beta$ is length ${ }^{-1}$. The characteristic length, $|\beta|^{-1}$, is the width of the mixing layer.

Nonlinear eigenvalue problem Eqs. (1), (3), (4) was solved numerically using an iteration procedure. At each iteration step, the system is being reduced to the Sturm-Liouville problem that was solved with the Prüfer method (a shooting method based on oscillation [1,9]). For a given $\beta$ of vortex line flow, $\beta_{3 D}$, one can choose $\beta$ of point vortex flow, $\beta_{2 D}$, in such a way that the velocity profiles are practically identical. This can be seen, for example, from Fig. 2, where the velocity profiles and the stream function are shown for $\beta_{3 D}=-1.0000$ and $\beta_{2 D}=-1.1374$. The values of $\beta_{2 D}$ and $\beta_{3 D}$ for which the average velocity profiles coincide form a curve in the plane $\left\{\beta_{2 D}, \beta_{3 D}\right\}$ shown in Fig. 3. This curve was found in the following way. For each $\beta_{3 D}$, we seek $\beta_{2 D}$ by the minimization of the sum

$$
\sum_{i=1}^{N} w_{i}\left|u_{i}^{2 D}-u_{i}^{3 D}\right|,
$$

where $u_{2 D}$ and $u_{3 D}$ are velocities in $2 D$ and $3 D$ problems, respectively, $N$ is the number of the mesh points. The weight coefficients, $w_{i}$, depend on the density of the mesh and the gradient of the velocity profile $u$. The distance between the walls, $h$, was chosen large enough for the solution to be applicable.

The parameters $\beta_{2 D}$ and $\beta_{3 D}$ have the meaning of the inverse temperature of two-dimensional and threedimensional motions. The fact, that the same velocity profile corresponds to different $\beta_{2 D}$ and $\beta_{3 D}$, indicates that the corresponding temperatures of two-dimensional and three-dimensional motions are different. The temperature of two-dimensional motion has a simple physical meaning: this is an average area bounded by 
the vortex trajectory. The area has orientation. Accordingly, temperature may have both signs. Negative temperature of the flow considered corresponds to clockwise pass of the curls of the vortex trajectories [2]. The physical meaning of temperature of three-dimensional vortex line motion is not known; therefore, a physical interpretation of the graph $\beta_{2 D}\left(\beta_{3 D}\right)$ is yet to be established. The result obtained seems an indication that for the shear flow between parallel walls three-dimensionality does not play an important role in formation of the averaged velocity profiles.

This paper has been published previously in the Russian journal [10] that is not distributed in the West and on internet.

Open Access This article is distributed under the terms of the Creative Commons Attribution License which permits any use, distribution, and reproduction in any medium, provided the original author(s) and the source are credited.

\section{References}

1. Akulenko, L., Nesterov, S.: High-precision Methods in Eigenvalue, Problems and Their Applications. Chapman \& Hall/CRC, Boca Raton (2005)

2. Berdichevsky, V.: Thermodynamics of Chaos and Order. Addison-Wesly-Longman, London (1997)

3. Berdichevsky, V.: Statistical mechanics of vortex lines. Phys. Rev. E. 57, 2885 (1998)

4. Berdichevsky, V.: On statistical mechanics of vortex lines. Int. J. Eng. Sci. 40, 123 (2002)

5. Berdichevsky, V.: Averaged equations of ideal fluid turbulence. Continuum Mech. Thermodyn. 19, 133 (2007)

6. Lions, P.-L., Majda, A.: Equilibrium statistical theory for nearly parallel vortex filaments. Commun. Pure Appl. Math. 53, $76(2000)$

7. Montgomery, D., Joyce, G.: Negative temperature states for 2-dimensional guiding-center plasma. J. Plasma Phys. 10, 107 (1973)

8. Montgomery, D., Joyce, G.: Statistical mechanics of "negative temperature" states. Phys. Fluids 17, 1139 (1974)

9. Pryce, J.: Numerical Solution of Sturm-Liouville Problems. Oxford University Press, New York (1994)

10. Shirkov, L., Berdichevsky, V.: Izvestiya Vuzov. Severo-Kavkazkii Region, Special Issue Actual problems of mathematical hydrodynamics (2009) 